OPEN ACCESS

Edited by:

Bernd Rehm,

Griffith University, Australia

Reviewed by:

Takeharu Tsuge

Tokyo Institute of Technology, Japan

Christopher John Brigham,

Wentworth Institute of Technology,

United States

Ignacio Poblete-Castro,

Andres Bello University, Chile

*Correspondence:

Qian Wang

qiqi20011983@gmail.com

${ }^{\dagger}$ These authors have contributed equally to this work

Specialty section:

This article was submitted to Industrial Biotechnology,

a section of the journal

Frontiers in Bioengineering and

Biotechnology

Received: 04 December 2019 Accepted: 12 August 2020

Published: 27 August 2020

Citation:

Li Y, Sun Z, Xu Y, Luan Y, Xu J, Liang Q, Qi Q and Wang Q (2020)

Enhancing the Glucose Flux of an

Engineered EP-Bifido Pathway

for High Poly(Hydroxybutyrate) Yield

Production

Front. Bioeng. Biotechnol. 8:517336.

doi: 10.3389/fbioe.2020.517336

\section{Enhancing the Glucose Flux of an Engineered EP-Bifido Pathway for High Poly(Hydroxybutyrate) Yield Production}

\author{
Ying Li1t, Zhijie Sun't, Ya Xu', Yaqi Luan', Jiasheng Xu', Quanfeng Liang', \\ Qingsheng Qi ${ }^{1,3}$ and Qian Wang ${ }^{1 *}$
}

1 National Glycoengineering Research Center, State Key Laboratory of Microbial Technology, Shandong University, Qingdao, China, ${ }^{2}$ Marine Biology Institute, Shantou University, Shantou, China, ${ }^{3}$ CAS Key Lab of Biobased Materials, Qingdao Institute of Bioenergy and Bioprocess Technology, Chinese Academy of Sciences, Qingdao, China

Background: As the greenhouse effect becomes more serious and carbon dioxide emissions continue rise, the application prospects of carbon sequestration or carbonsaving pathways increase. Previously, we constructed an EP-bifido pathway in Escherichia coli by combining Embden-Meyerhof-Parnas pathway, pentose phosphate pathway and "bifid shunt" for high acetyl-CoA production. There is much room for improvement in the EP-bifido pathway, including in production of target compounds such as poly(hydroxybutyrate) (PHB).

Result: To optimize the EP-bifido pathway and obtain higher PHB yields, we knocked out the specific phosphoenolpyruvate phosphate transferase system (PTS) component II Cglc, encoded by ptsG. This severely inhibited the growth and sugar consumption of the bacterial cells. Subsequently, we used multiple automated genome engineering (MAGE) to optimize the ribosome binding site (RBS) sequences of galP (galactose: $\mathrm{H}$ (+) symporter) and glk (glucokinase gene bank: NC_017262.1), encoding galactose permease and glucokinase, respectively. Growth and glucose uptake were partially restored in the bacteria. Finally, we introduced the glf (UDP-galactopyranose) from Zymomonas mobilis mutase sugar transport vector into the host strain genome.

Conclusion: After optimizing RBS of galP, the resulting strain L-6 obtained a PHB yield of $71.9 \%(\mathrm{~mol} / \mathrm{mol})$ and a $76 \mathrm{wt} \%$ PHB content using glucose as the carbon source. Then when glf was integrated into the genome strain $L-6$, the resulting strain M-6 reached a $5.81 \mathrm{~g} / \mathrm{L}$ PHB titer and $85.1 \mathrm{wt} \%$ PHB content.

Keywords: poly(hydroxybutyrate) yield, glucose flux, EP-bifido pathway, MAGE, Escherichia coli

\section{INTRODUCTION}

In 2018, global carbon dioxide emissions increased $1.7 \%$ over the previous year, hitting a record high of 33.143 billion tons. Accelerating the adoption of renewable energy and improving energy efficiency in response to global warming are urgent priorities (International Energy Agency, 2019). Bio-manufacturing, which uses food crops as raw materials, has wide application prospects. 
Through biological manufacturing, biomass resources can be converted to ethanol, polylactic acid, 1, 3-propanediol, and other bulk chemicals (Ragauskas et al., 2006; Lee et al., 2012). A key barrier to this process is the $\mathrm{CO}_{2}$ emissions that occur during natural aerobic fermentation. Many carbon dioxide fixation pathways have been exploited using the six carbon fixation pathways discovered in nature (Gong et al., 2016). However, complex reaction steps and enzyme requirements limit the broad application of these carbon sequestration pathways (Erb et al., 2007; Schwander et al., 2016).

In natural microorganism fermentation, glucose can be transformed through the Embden-Meyerhof-Parnas (EMP) pathway to pyruvate. Pyruvate metabolism leads to the production of two molecules of acetyl-CoA (AcCoA), the key precursor of ethanol, butanol, fatty acids, amino acids, and pharmaceuticals. This process produces two molecules of $\mathrm{CO}_{2}$ from one mol of glucose, making it an uneconomical way to biosynthesize products using AcCoA as precursor. Other glycol metabolism pathways, such as the phosphoketolase pathway, employ a pentose phosphate specific transketolase to produce a mixture of ethanol, lactic acid, and $\mathrm{CO}_{2}$. Additionally, the bifido bacteria exclusive bifid shunt pathway can generate $1 \mathrm{~mol}$ of lactic acid and $1.5 \mathrm{~mol}$ of acetate from $1 \mathrm{~mol}$ of glucose (Meile et al., 2001; Posthuma et al., 2002). However, all of these glucose metabolic pathways lose carbon in the form of $\mathrm{CO}_{2}$ during the decarboxylation process. Therefore, several carbon-saving pathways, including the non-oxidative glycolytic (NOG) and EPbifido pathways, have been engineered. The NOG pathway can transform all six carbon atoms of glucose into three AcCoA molecules without $\mathrm{CO}_{2}$ loss (Bogorad et al., 2013). However, it cannot provide the reducing power NADPH that is needed for $\mathrm{PHB}$ and other chemicals biosynthesis. The EP-bifido pathway employs EMP, pentose phosphate pathway (PPP) and the Bifido shunt for high-yield of AcCoA generation. As a reducing power sponsor, the oxidation part of the PPP consumes $1 \mathrm{~mol}$ of glucose, and provides $2 \mathrm{~mol}$ of NADPH and $1 \mathrm{~mol}$ of xylulose-5-phosphate (X5P). The enzyme encoded by the $f / x p k$ gene of the EP-bifido pathway has both fructose-6-phosphate (F6P) and X5P activity. It is able to catalyze $\mathrm{X} 5 \mathrm{P}$ to form acetyl phosphate (AcP) and glyceraldehyde 3-phosphate (G3P) or split F6P to form erythrose 4-phosphate and AcP. The former G3P can generate AcP through carbon rearrangement, each of these processes releases only $1 \mathrm{~mol}$ of $\mathrm{CO}_{2}$, thus saving the carbon source to a certain extent (Figure 1). This carbon-saving pathway has been applied to the production of several compounds that use AcCoA as the precursor (Wang et al., 2019). Previously, we achieved a relatively high level of production and yield, but there remains room to improve carbon conversion in our system.

In Escherichia coli, glucose is transported through the phosphate transferase system (PTS). This system is involved in phosphoenolpyruvate (PEP)-dependent sugar transport and its activity has an important impact on carbon flux redistribution in the PEP and pyruvate nodes (Gosset, 2005). Glucose was phosphorylated to G6P by the phosphoryl generated from PEP, which was dephosphorylated to form pyruvate. Then pyruvate further decarboxylated to $\mathrm{AcCoA}$ and released $1 \mathrm{~mol}$ of $\mathrm{CO}_{2}$, leading to the loss of the carbon source. In addition, PEP is a key intermediate metabolite of the EMP pathway. Therefore, an increase in the EMP pathway reduces the carbon conversion efficiency of the EP-bifido pathway.

Poly(hydroxybutyrate) (PHB) is the most common poly(hydroxyalkanoate) (PHA). PHB can be synthesized and accumulated by more than 300 microorganisms as both an energy and carbon store (Lee and Choi, 2001; van der Walle et al., 2001). The in vivo biosynthesis of $\mathrm{PHB}$ requires three steps using $\mathrm{AcCoA}$ as the precursor, and $\mathrm{PHB}$ production has been intensively studied (Lee et al., 1994; Wang Q. et al., 2009). Many strategies have been applied to engineer E. coli to improve PHB production, however, the yield still has much room to progress. By overexpressing NAD kinase, recombinant E. coli produced $14 \mathrm{~g} / \mathrm{L}$ PHB and the yield based on glucose reached $0.31 \mathrm{~mol} / \mathrm{mol}$ (0.15 g PHB/g glucose) (Li et al., 2009). By applying fed-batch strategy, E. coli could accumulate $125 \mathrm{~g} / \mathrm{L} \mathrm{PHB}$, but the yield based on glucose was only $0.46 \mathrm{~mol} / \mathrm{mol}$ ( $0.22 \mathrm{~g}$ PHB/g glucose) (Mozumder et al., 2014). Previously, we have achieved relatively high level of PHB content and PHB yield (68.4 wt\% and 63.7\% $\mathrm{mol} / \mathrm{mol}$, respectively) (Wang et al., 2019). In this study, we optimized the EP-bifido pathway for improved PHB production in E. coli. The non-PTS glucose transport pathway genes glk, galP and heterogeneous $g l f$ were enhanced and introduced into EPbifido strains through multiple automated genome engineering (MAGE) and conditional-replication, integration, excision, and retrieval (CRIM) plasmids. The improved PHB production indicates that our modification increased the efficiency of artificial carbon-saving pathways for high carbon conversion rate from glucose.

\section{MATERIALS AND METHODS}

\section{Culture Media and Conditions}

For plasmid preparation, E. coli strains were cultured at $37^{\circ} \mathrm{C}$ on a rotary shaker $(220 \mathrm{rpm})$ in test. For plasmid preparation, E. coli strains were cultured at $37^{\circ} \mathrm{C}$ on a rotary shaker $(220 \mathrm{rpm})$ in test tubes containing $5 \mathrm{~mL}$ Lysogeny broth (LB) medium. For PHB biosynthesis, $50-\mathrm{mL}$ shake flask cultures were started by $2 \%$ inoculation from the $5-\mathrm{mL}$ LB culture. The $50-\mathrm{mL}$ cultures contained M9 minimal medium with $0.2 \%$ yeast extract containing $20 \mathrm{~g} / \mathrm{L}$ glucose and shaken at $37^{\circ} \mathrm{C}$ in a rotary shaker $(120 \mathrm{rpm})$ for $48 \mathrm{~h}$. Overnight cultures were shaken at $37^{\circ} \mathrm{C}$ in a rotary shaker $(220 \mathrm{rpm})$. Antibiotics were added as follows: ampicillin (Amp) $100 \mu \mathrm{g} / \mathrm{mL}$, spectinomycin (Spc) $50 \mu \mathrm{g} / \mathrm{mL}$, and chloromycetin $(\mathrm{Cm}) 25 \mu \mathrm{g} / \mathrm{mL}$. For MAGE procedure, strains were cultivated in SOB medium.

Lysogeny broth medium contains (g/L): tryptone (10), yeast extract (5), and $\mathrm{NaCl}(10)$. $\mathrm{M} 9$ medium contains (g/L): $\mathrm{Na}_{2} \mathrm{HPO}_{4} \cdot 12 \mathrm{H}_{2} \mathrm{O}$ (15.138), $\mathrm{KH}_{2} \mathrm{PO}_{4}$ (3), $\mathrm{NaCl}(0.5)$, and $\mathrm{NH}_{4} \mathrm{Cl}$ (1). SOB medium contains (g/L): tryptone (20), yeast extract (5), and $\mathrm{NaCl}$ (5).

\section{Strains and Plasmids}

All E. coli strains and plasmids used are listed in Table $\mathbf{1}$. DH-EP was used as the starting strain for further genetic 


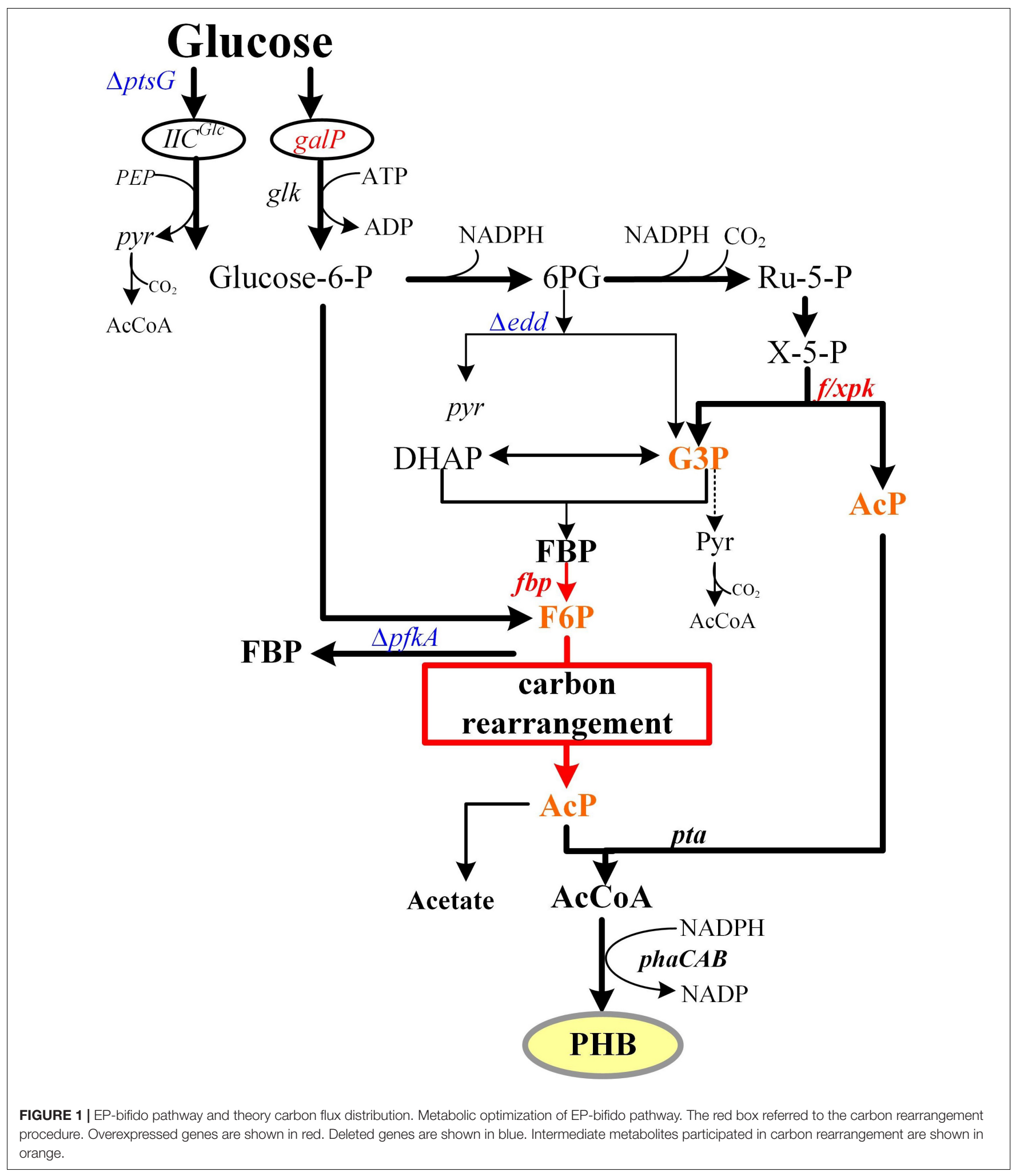

manipulation. All primers used for molecular manipulations are listed in Table 2.

The Red homologous recombination method was employed for gene deletion. The pTKRED complementary plasmid was transformed into the target strain. Deletion fragments of $p t s G$ gene were amplified from the JW1087-2 single-gene knockout mutant (Baba et al., 2006) (bought from Coli Genetic Stock Center, CGSC) using primers Q-ptsG-F/Q-ptsG-R. 
TABLE 1 | Bacterial strains and plasmids used in this study.

\begin{tabular}{|c|c|c|}
\hline $\begin{array}{l}\text { Strain and } \\
\text { plasmids }\end{array}$ & Relevant properties & Sources \\
\hline \multicolumn{3}{|l|}{ Strains } \\
\hline JW1087-2 & JW25113 derivative, $\Delta$ ptsG:kan & Baba et al. (2006) \\
\hline DH-EP & $\mathrm{DH} 5 \alpha$ derivative, $\Delta$ edd $\Delta p f k A$ & Wang et al. (2019) \\
\hline DH-EPP & $\mathrm{DH} 5 \alpha$ derivative, $\Delta$ edd $\Delta p f k A \Delta p t s G$ & This study \\
\hline L-6 & $\begin{array}{l}\text { DH-EPP derivative, } \Delta e d d \Delta p f k A \Delta p t s G \text { galP } \\
\text { RBS:TGAAAGGGAAA }\end{array}$ & This study \\
\hline M-6 & $\begin{array}{l}\text { L-6 derivative, } \\
\Delta \text { edd } \Delta p f k A \Delta p t s G: t r c-r b s-g l f_{z m}\end{array}$ & This study \\
\hline \multicolumn{3}{|l|}{ Plasmids } \\
\hline pCAB & $\begin{array}{l}\text { pBluescriptll SK, phbC and phbAB gene } \\
\text { from Ralstonia eutropha }\end{array}$ & Wang et al. (2019) \\
\hline pCDFtrc & Cloning vector, Spe ${ }^{R}$ & Wang et al. (2019) \\
\hline pFF & $\begin{array}{l}\text { pCDFtrc, fxpk gene from } B \text {. adolescentis } \\
\text { and fbp gene from } E \text {. coli }\end{array}$ & Wang et al. (2019) \\
\hline pKD3 & $\begin{array}{l}\text { Template plasmid with } \mathrm{Cm}^{\mathrm{R}} \text { gene and FLP } \\
\text { recognition target }\end{array}$ & $\begin{array}{l}\text { Datsenko and } \\
\text { Wanner (2000) }\end{array}$ \\
\hline pTKRED & $\mathrm{P}_{\mathrm{BAD}}$ promoter containing plasmid, Spe $\mathrm{R}^{\mathrm{R}}$ & $\begin{array}{l}\text { Kuhlman and Cox } \\
\text { (2010) }\end{array}$ \\
\hline pCP20 & $\begin{array}{l}\text { Helper plasmid expressing FLP } \\
\text { recombinase, ts-rep, } \mathrm{Amp}^{R}, \mathrm{Cm}^{R}\end{array}$ & $\begin{array}{l}\text { Datsenko and } \\
\text { Wanner (2000) }\end{array}$ \\
\hline pAH69 & $\begin{array}{l}\text { Helper plasmid expressing } \mathrm{HKO} 22 \\
\text { integrase, } \mathrm{Amp}^{\mathrm{R}}\end{array}$ & $\begin{array}{l}\text { Haldimann and } \\
\text { Wanner (2001) }\end{array}$ \\
\hline
\end{tabular}

TABLE 2 | Key oligonucleotide primers used in this study for DNA manipulation.

\begin{tabular}{ll}
\hline Primers & Sequence $\left(\mathbf{5}^{\prime} \mathbf{- 3} \mathbf{3}^{\prime}\right)$ \\
\hline Q-ptsG-F & 5'-GGCTGTGTTGAAAGGTGTTGC-3' \\
Q-ptsG-R & 5'-AACGCGCTATATTGCAGAGG-3' \\
Glf-F & 5'-GGTCGGTAAATCGCTGCTTGACAATTAATCATCCGGC \\
& TCGTATAATGTCTAGAGAAAGAGGAGAAATACTAGATGAG \\
& TCTGAAAGTAGTCAGGGTC-3' \\
Glf-R & 5'-GCCTACCCGGATATATCGTGAGGATGCGAATTGTG \\
& TAGGCTGGAGCTGCTTC-3' \\
R6K-F & 5'-TCGCATCCTCACGATAATATCCGGGTAGGC-3' \\
R6K-R & 5'-TTGTCAAGCAGCATCAGCGATTACCGACCGATCC \\
& GGCCACGATGCGTCC-3' \\
\hline
\end{tabular}

\section{Measurement of Extracellular Metabolites}

A spectrophotometer was used to measure the optical density at $600 \mathrm{~nm}\left(\mathrm{OD}_{600}\right)$ of the bacterial culture. PHB was quantified using gas chromatography (GC). Cells were harvested by centrifugation at $6,000 \times g$ for $10 \mathrm{~min}, 4^{\circ} \mathrm{C}$. The cell pellets were washed twice with distilled water and lyophilized for $7 \mathrm{~h}$. Before GC analysis, $1 \mathrm{~mL}$ chloroform, $850 \mu \mathrm{L}$ methanol, and $150 \mu \mathrm{L}$ sulfuric acid $(98 \%, \mathrm{w} / \mathrm{w})$ were added to the weighed cells in vials. The vials were incubated at $100^{\circ} \mathrm{C}$ for $1 \mathrm{~h}$. Then, $1 \mathrm{~mL}$ water was added for stratification and cooling vials. After standing for $1 \mathrm{~h}$, the mixture separated into layers and the heavier chloroform phase was transferred to new vial for GC analysis. The GC detection process was performed using a Shimadzu GC2010 gas chromatograph (Kyoto, Japan) equipped with an AOC-20i auto injector and a Restek Rtx-5 column. PHB standard samples of methyl-(R)-3-hydroxybutyrate (Sigma-Aldrich) were dissolved in chloroform and analyzed by GC. The temperature program used was: $80^{\circ} \mathrm{C}$ for $1 \mathrm{~min}$, ramped to $120^{\circ} \mathrm{C}$ at $10^{\circ} \mathrm{C} / \mathrm{min}$, then ramped to $160^{\circ} \mathrm{C}$ at $45^{\circ} \mathrm{C} / \mathrm{min}$ for $5 \mathrm{~min}$, and the total time was $10.89 \mathrm{~min}$.

For extracellular metabolite analysis, $1 \mathrm{~mL}$ of culture was centrifuged at $2,000 \times g$ for $2 \mathrm{~min}$. The supernatant was filtered through a $0.22-\mu \mathrm{m}$ syringe filter for high-performance liquid chromatography analysis. Glucose, acetate, and pyruvate were measured on an ion exchange column (HPX-87H; Bio-Rad Labs) with a differential refractive index detector (Shimadzu RID-10A). A $0.5-\mathrm{mL} / \mathrm{min}$ mobile phase using a $5-\mathrm{mM} \mathrm{H}_{2} \mathrm{SO}_{4}$ solution was applied to the column. The column was operated at $65^{\circ} \mathrm{C}$.

\section{MAGE Procedure}

The ribosome binding sites (RBSs) designed for the modulation of GalP and glk transcription rates were $5^{\prime}$-GTCGTACTC ACCTATCTTAATTCACAATAAAAAATAACCADDRRRRRD DDDATCATGCCTGACGCTAAAAAACAGGGGCGGTCAAA CAAG-3' $(\mathrm{D}=\mathrm{A}, \mathrm{G}, \mathrm{T}$; and $\mathrm{R}=\mathrm{A}, \mathrm{G})$ and 5'-GCCGCCCACA TCACCGACTAATGCATACTTTGTCATTCTHHHHYYYYYH HGCTAAAGTCAAAATAATTCTTTCTCACACTGTAAATAC CT-3' $(H=T, C, A$; and $Y=T, C)$, respectively, with four phosphorothioated bases at the $5^{\prime}$ terminus. The initiation of MAGE requires that pTKRED was transformed into the target strain. The MAGE cycles were performed by growing DH-EPP in $5 \mathrm{~mL} \mathrm{SOB}$ medium at $30^{\circ} \mathrm{C}$ and shaking at $220 \mathrm{rpm}$ for $12 \mathrm{~h}$. For the first MAGE round, 5-mL shake flask cultures using SOB broth were started with a $1 \%$ inoculation from the overnight culture. Isopropyl- $\beta$-D-thiogalactopyranoside (IPTG) was added to a final concentration of $0.5 \mathrm{mM}$ to induce $\lambda$-prophage (bet, gam, and exo) gene expression. Cells were then incubated at $30^{\circ} \mathrm{C}$ and shaking at $220 \mathrm{rpm}$ until reaching an $\mathrm{OD}_{600}$ of 0.5 to 0.6. Cells were collected $(2 \mathrm{~mL})$, pelleted, and washed three times with cold sterile water to make them electrocompetent. ssDNA mixture $(1 \mu \mathrm{M})$ was added to electrocompetent cells and electroporated at $2.5 \mathrm{kV}$. To start the second MAGE round, cells were recovered in $5 \mathrm{~mL}$ SOC with IPTG until their $\mathrm{OD}_{600}$ reached 0.5 to 0.6 , after which cells underwent pelleting, washing, and electroporation. Three to four MAGE rounds were performed per day and 16 cycles were performed in total. The resulting pool of variants were then characterized using the Nile red assay.

\section{Screening of PHB Competent Cells by Nile Red Assay}

When PHB is combined with Nile red dye a red color is produced. We transformed the $\mathrm{PCAB}$ plasmid into these variants and added $100 \mu \mathrm{L}$ Amp, $50 \mu \mathrm{L}$ IPTG, $20 \mathrm{~g} / \mathrm{L}$ glucose, and $200 \mu \mathrm{L}$ Nile red dye to the solid M9 medium supplemented with $0.2 \%$ yeast extract. The MAGE variants were diluted 200 -fold and spread onto several plates. After incubation at $37^{\circ} \mathrm{C}$ for $16 \mathrm{~h}$, the plates were placed at $4^{\circ} \mathrm{C}$ for 3 days to allow the color reaction to develop. Based on the color difference, we picked single red colonies for sequencing. For all the sequenced colonies with mutations identified, the pTKRED plasmid was removed and the strains were transformed with the $\mathrm{pFF}$ and $\mathrm{pCAB}$ plasmids for further verification of the $\mathrm{PHB}$ competent cells. 


\section{Integration of glf}

For glf integration, the trc-rbs-glf module was amplified from Zymomonas mobilis genomic DNA by PCR using primers GLF$\mathrm{F}$ and GLF-R. The PCR product (trc-rbs-glf) was cloned into a vector that carries $\mathrm{R} 6 \mathrm{~K}$ replicon and phage attachment sites (attP). This plasmid was named R6K-glf and was confirmed by DNA sequencing. The pAH69 helper plasmid was transformed into the L-6 strain. The target strain carrying pAH69 was incubated overnight at $30^{\circ} \mathrm{C}$ and transferred to $37^{\circ} \mathrm{C}$ for $1 \mathrm{~h}$ before transfection. Then the pR6K-glf plasmid was introduced into L- 6 by electroporation. The centrifuged bacteria were plated onto plates containing $25 \mu \mathrm{g} / \mathrm{mL}$ kanamycin for overnight incubation at $37^{\circ} \mathrm{C}$. R6K-glf positive transformants were selected by their $\mathrm{kan}^{\mathrm{R}}$ phenotype and were verified by PCR.

\section{Determination of $\mathrm{CO}_{2}$ Emissions}

$\mathrm{CO}_{2}$ emission was determined using a thermostatic oscillation incubator with a $\mathrm{CO}_{2}$ detector (BCP- $\mathrm{CO}_{2}$, Bluesens, Germany) that monitored $\mathrm{CO}_{2}$ volume every $20 \mathrm{~s}$ and transmitted the data to a computer. Cultures were grown at $37^{\circ} \mathrm{C}$ with shaking at $150 \mathrm{rpm}$.

\section{RESULTS}

\section{Inhibiting PTS to Reduce PEP Consumption}

In E. coli, glucose is transported through the PTS system. This system is involved in PEP-dependent sugar transport and its activity has an important impact on carbon flux redistribution in the PEP and pyruvate nodes (Gosset, 2005). Glucose transport into the cytoplasm by $\mathrm{EIICB}^{\mathrm{Glc}}$ (encoded by $p t s G$ ) is coupled to its phosphorylation. The phosphate group is derived from PEP and is transferred via a cascade of proteins, enzyme I (EI), HPr, EIIA, and EIIB. This procedure consumes almost half of the PEP (Valle et al., 1996; Wang et al., 2012). Glucose was phosphorylated to G6P by phosphoryl generated from PEP dephosphorylated to pyruvate, the formed pyruvate is further decarboxylated to AcCoA and releases $1 \mathrm{~mol} \mathrm{CO}_{2}$, leading to carbon source loss. While PEP is not the precursor in our study, PEP consumption would convert carbon flux to the EMP pathway, which is not desirable in our EP-bifido pathway. Therefore, modulation of the PEP-independent uptake and phosphorylation system is required. Knocking out $p t s G$ and replacing it with other glucose transport pathways is a common method used in the production of PEP-precursor products (Gosset, 2005; Lee et al., 2005; Li et al., 2013; Kyselova et al., 2018). Therefore, we knocked out $p t s G$ in strain DH-EP and named the strain DH-EPP. But found that $p t s G$ deletion severely impaired the growth capacity of the resulting strain. Compared with DH-EP (pFFpCAB) strain, PHB yield of DH-EPP strain decreased from 63.7 to $26.3 \%$ ( $\mathrm{mol} / \mathrm{mol}$ ) (Figure 2).

For glucose uptake recovery, replacing PTS with an alternative PEP-independent uptake and phosphorylation system could be an efficient solution to this problem.

\section{Improving Glucose Flux Through Non-PTS Pathway}

When E. coli strains lack PTS, the low affinity galactose: $\mathrm{H}^{+}$symporter, GalP, encoded by galP, is induced. Glucose

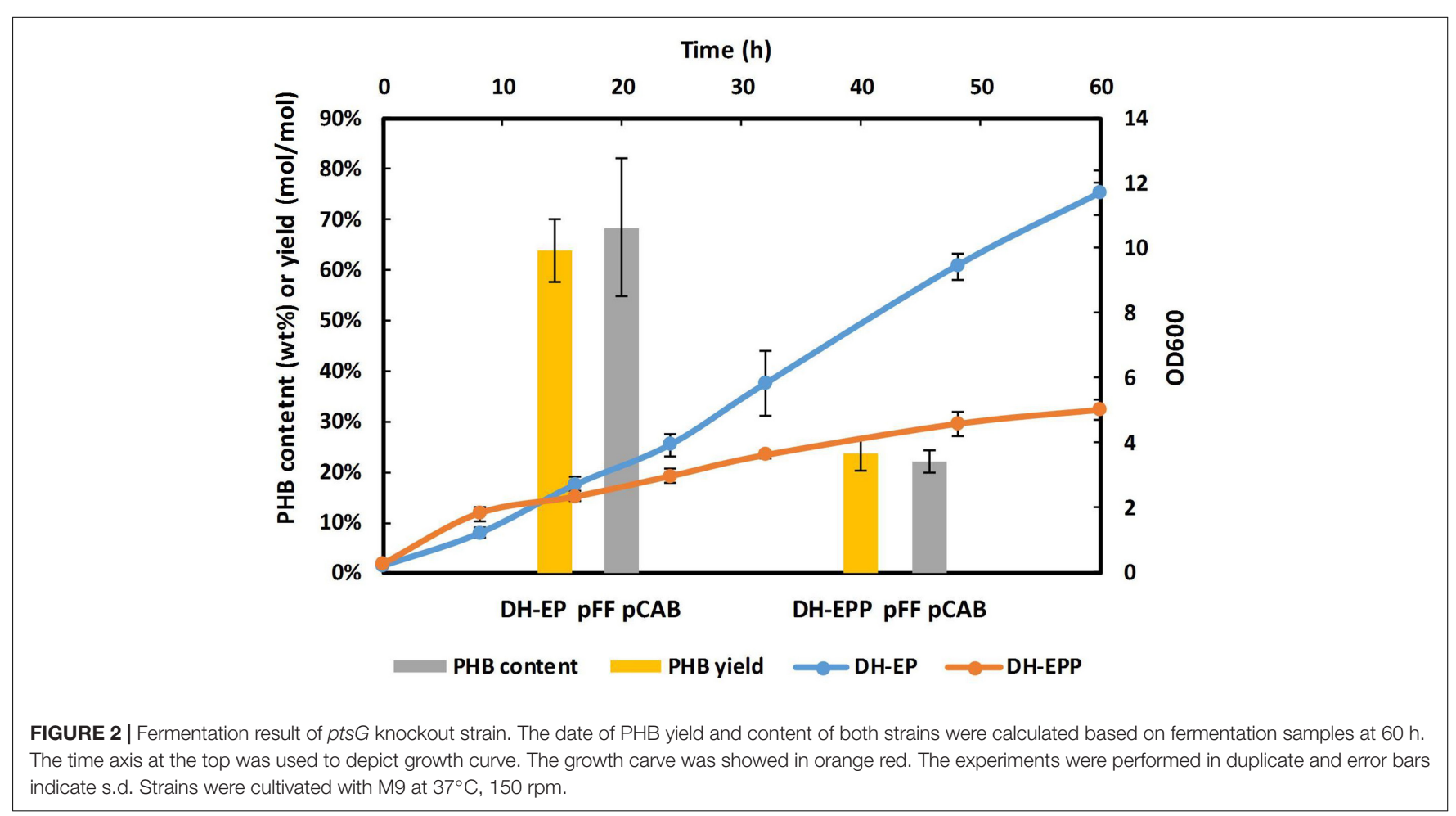




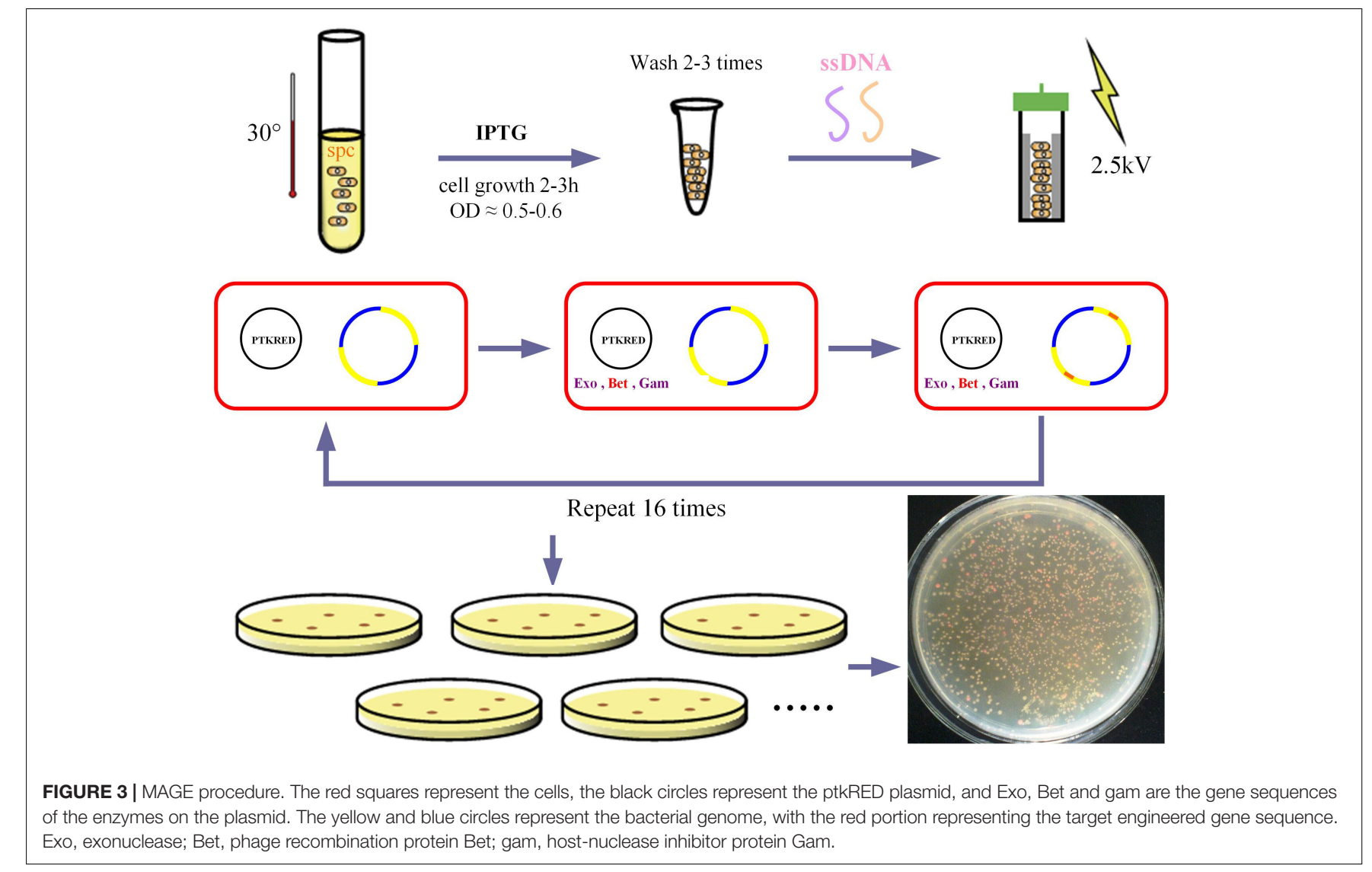

internalized by GalP must be phosphorylated by glucokinase (encoded by $g l k$ ), which catalyzes the ATP-dependent phosphorylation of glucose to generate G6P in the cytoplasm without $\mathrm{CO}_{2}$ emission, thus saving the carbon source (Gosset, 2005). Therefore, we modified galP and glk expression levels to improve the glucose utilization rate. While increasing galP and $g l k$ transcriptional levels through plasmid overexpression is a pervasive strategy (Hernandez-Montalvo et al., 2003; Wang et al., 2006), it has several disadvantages, including metabolic burden and unexpected lateral effects. Modulation of galP and $g l k$ expression levels through plasmid overexpression or high strength promoter substitution cannot provide multiple combination of expression intensity for screening. Discovering a way to effectively modulate the transcription of the two genes to an optimal strength in combination is a pressing problem. We adopted MAGE to simultaneously regulate galP and glk expression levels (Wang H.H. et al., 2009). Using this approach, colonies with high PHB yields can be identified using Nile red dye staining.

After 16 rounds of MAGE modulation (Figure 3), recombinant strains were screened by Nile red staining. Recombinant strains with higher PHB production showed redder color. Screening and sequencing results are shown in Table 3. Using single colony color screening, we found that the RBS of both glk and galP genes were changed, and the amount of ssDNA (single string DNA) recombination of glk exceeded that of galP. This may be because the location of the glk gene is more susceptible to ssDNA recombination during genome replication. However, no recombinant was screened out in which the two genes were simultaneously mutated.

TABLE 3 | Screening result of MAGE recombination strains.

\begin{tabular}{|c|c|c|c|}
\hline Strain number & Gene & Origin RBS sequence & Mutated RBS sequence \\
\hline 1 & $g / k$ & GGAGCAGTTGA & GAAGGGAGAGG \\
\hline 2 & $g / k$ & GGAGCAGTTGA & GAAAGAAATGA \\
\hline 6 & galP & TATTGGAGGGC & TGAAAGGGAAA \\
\hline 9 & $g \mid k$ & GGAGCAGTTGA & GGAGGGATGGA \\
\hline 16 & $g / k$ & GGAGCAGTTGA & TAGGAGGAGTT \\
\hline 18 & $g / k$ & GGAGCAGTTGA & AAAAAGGGTTA \\
\hline 19 & $g / k$ & GGAGCAGTTGA & GAAGGAGGGGT \\
\hline 20 & galP & TATTGGAGGGC & GGAGAGGGTTA \\
\hline 21 & $g / k$ & GGAGCAGTTGA & TGGGGGGAGGG \\
\hline 22 & $g / k$ & GGAGCAGTTGA & AAAGGGGTTTG \\
\hline 23 & $g / k$ & GGAGCAGTTGA & GAAGGGGTTTG \\
\hline 24 & $g / k$ & GGAGCAGTTGA & AGAGGAAGAGA \\
\hline 27 & $g / k$ & GGAGCAGTTGA & AAAAGGGATAG \\
\hline 28 & $g / k$ & GGAGCAGTTGA & TTGGAAGATAT \\
\hline 30 & $g / k$ & GGAGCAGTTGA & TGAGGAATGAA \\
\hline 32 & $g / k$ & GGAGCAGTTGA & GTGGAAATAGA \\
\hline 36 & $g / k$ & GGAGCAGTTGA & TTAGGGGGAGT \\
\hline 51 & $g / k$ & GGAGCAGTTGA & TTAAGGGATAT \\
\hline 62 & $g / k$ & GGAGCAGTTGA & GGAAGGAGAAT \\
\hline
\end{tabular}


Nineteen mutant strains were selected and transformed with pFF and pCAB, subsequently. Three batches fermentation of these 19 strains lead to the selection of strains in consideration of glucose consumption and PHB content, named EPPG-6,
EPPG-16, EPPG-51, and EPPG-62 (Figure 4D). Then, we repeated fermentation using the four selected strains (Figure 4E). Fermentation results showed that after glucose transport system modulation, the DH-EPPG-6 strain had a recovered growth rate.

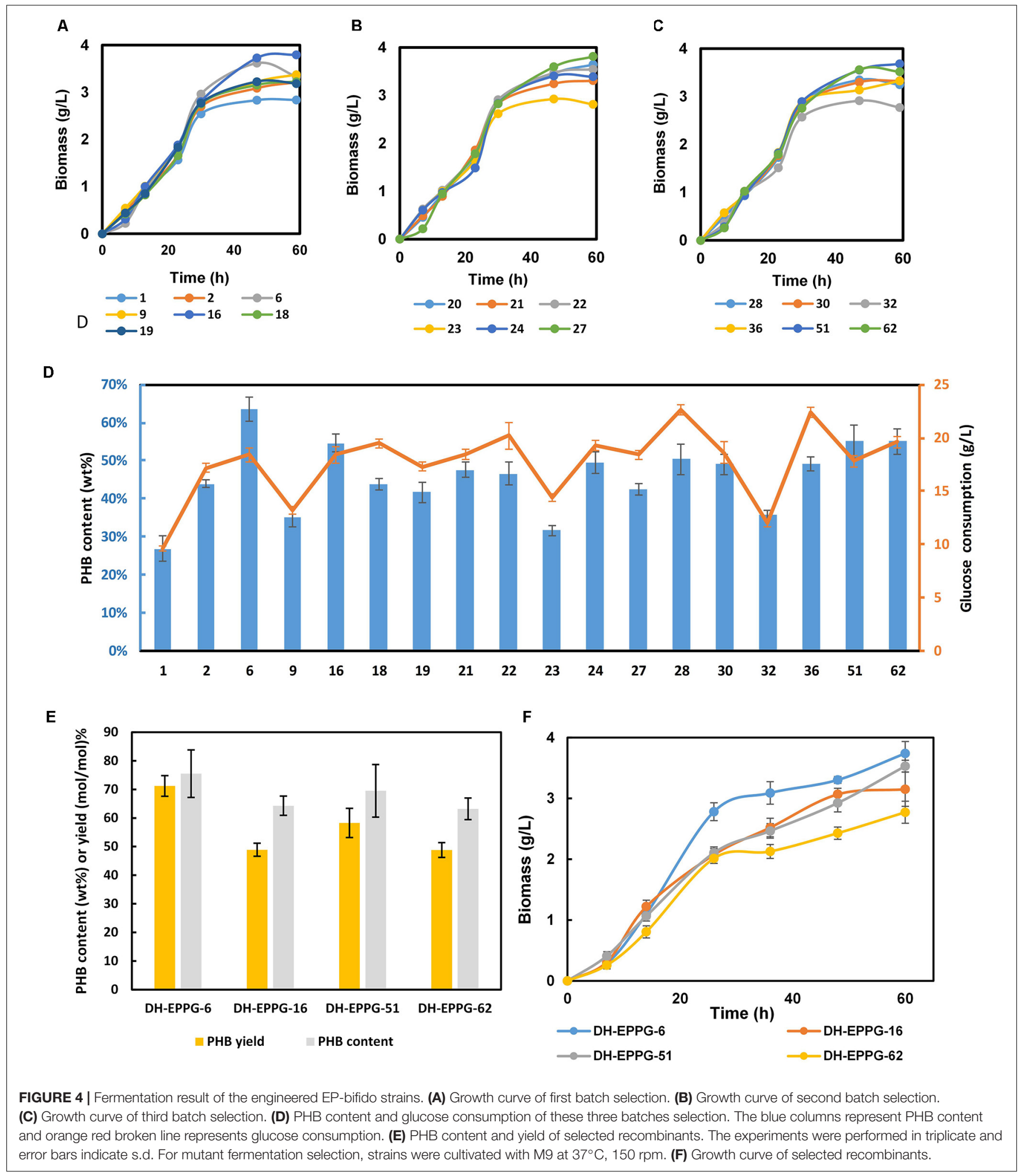


The PHB yield reached $71.2 \%(\mathrm{~mol} / \mathrm{mol})$, which was $63.7 \%$ higher than that of the strain DH-EP. Then DH-EPPG-6 strain was renamed L-6, in which the wild type RBS sequence of galP was mutated to TGAAAGGGAAA.

\section{Expressing Heterogeneous Sugar Transporter to Reinforce Glucose Uptake}

To further enhance the consumption of glucose, we compared the kinetic parameters of several transporters with their glucose transport capacity and energy consumption during glucose internalization and phosphorylation (Gosset, 2005). Because the transmembrane proton potential is a form of energy, the energy consumption of the glf sugar transporter from Zymomonas mobilis is comparable to that of the E. coli glucose-specific PTS (PTS ${ }^{\mathrm{Glc}}$ ). Compared with $g a l P$, the $g l f_{z m}$ transporter used less energy to produce a higher maximum velocity. Therefore, the high-rate, low-energy sugar transporter $g l f_{z m}$ was chosen to improve the glucose absorption capacity of engineered bacteria. Then $g l f_{z m}$ was inserted at the attP genomic site of strain L-6 using the CRIM plasmid system (Haldimann and Wanner, 2001), resulting the strain M-6. We deduced that $g l f$ integration was conducive to growth recovery in later growth stages. In Figure 5B, M-6 (pFFpCAB) showed a better growth
A

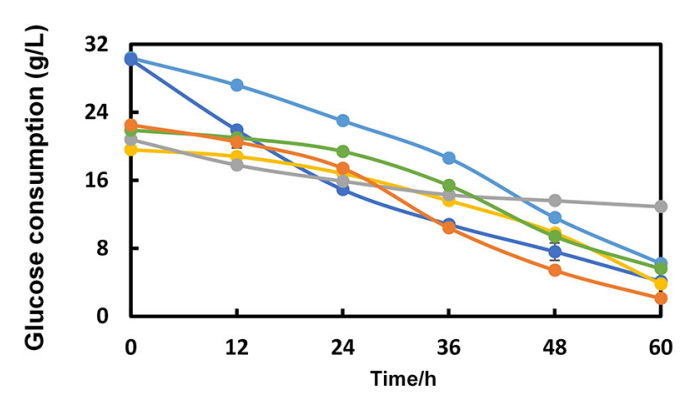

$\longrightarrow$ DH5 $\alpha$ pCDF pCAB $\rightarrow$ DH5 5 pFF pCAB $\longrightarrow$ DH-EP pFF pCAB

$\multimap$ DH-EPP pFF pCAB $\multimap$ L-6 pFF pCAB $\multimap-\mathrm{M}-6$ pFF pCAB

C

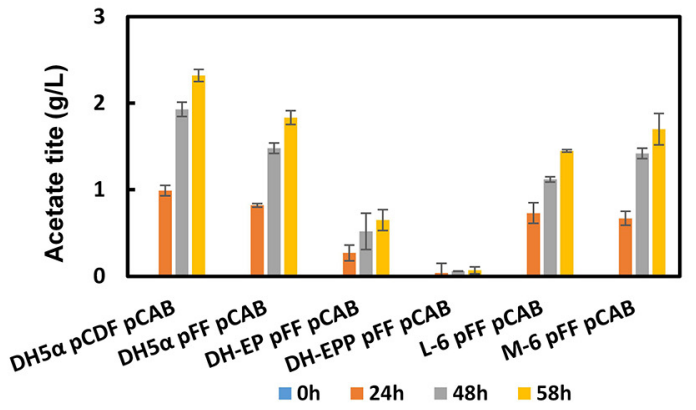

E

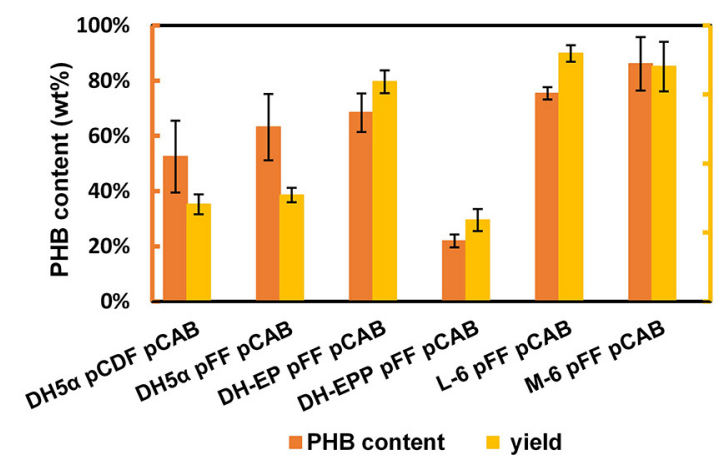

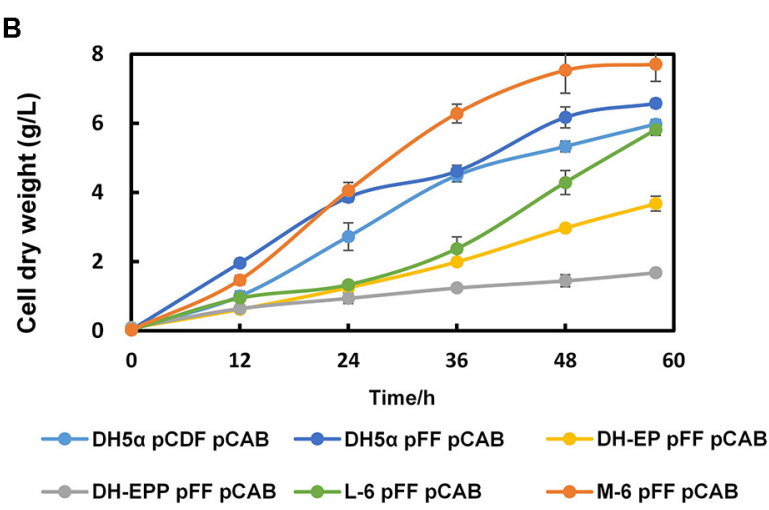

D

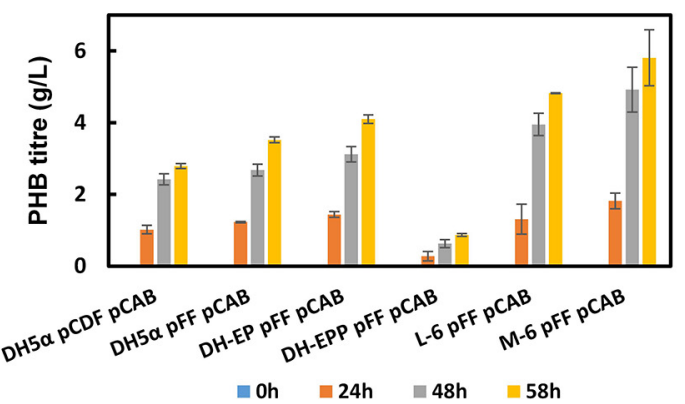

FIGURE $\mathbf{5}$ | The fermentation profile and PHB yield of all the engineered EP-bifido strains. (A) Glucose consumption. (B) Cell dry weight. (C) Acetate production. (D) PHB production. (E) PHB content and PHB yield. (F) Glucose consumption and acetate normalized by residual cell mass. The experiments were performed in triplicate and error bars indicate s.d. Strains were cultivated in $\mathrm{M} 9$ with glucose at $37^{\circ} \mathrm{C}, 150 \mathrm{rpm}$. 
TABLE 4 | Specific growth rates and glucose utilization rates of DH5 $\alpha$, DH-EP (pFFpCAB), DH-EPP (pFFpCAB), L-6 (pFFpCAB), and M-6 (pFFpCAB).

\begin{tabular}{|c|c|c|}
\hline Strains & $\begin{array}{l}\text { Specific growth } \\
\text { rate }\left(\mathrm{h}^{-1}\right)\end{array}$ & $\begin{array}{l}\text { Specific glucose utilization } \\
\text { rate }\left(\mathrm{g} \cdot \mathrm{L}^{-1} \cdot \mathrm{h}^{-1}\right)\end{array}$ \\
\hline $\mathrm{DH} 5 \alpha(\mathrm{pFFpCAB})$ & $0.35 \pm 0.011$ & $0.78 \pm 0.02$ \\
\hline DH-EP (pFFpCAB) & $0.20 \pm 0.020$ & $0.41 \pm 0.03$ \\
\hline DH-EPP (pFFpCAB) & $0.09 \pm 0.002$ & $0.23 \pm 0.01$ \\
\hline L-6 (pFFpCAB) & $0.28 \pm 0.018$ & $0.52 \pm 0.01$ \\
\hline M-6 (pFFpCAB) & $0.50 \pm 0.026$ & $0.71 \pm 0.03$ \\
\hline
\end{tabular}

Specific growth rates and glucose utilization rates were calculated based on the 0 $48 \mathrm{~h}$ data.

TABLE 5 | PHB productivity of the engineered PHB-producing strains.

\begin{tabular}{lc}
\hline Strains & PHB titer $\left(\mathbf{g} \cdot \mathbf{L}^{-\mathbf{1}} \cdot \mathbf{h}^{-\mathbf{1}}\right)$ \\
\hline DH5 $\alpha$ (pCDF pCAB) & $0.048 \pm 0.07$ \\
DH5 $\alpha$ (pFF pCAB) & $0.061 \pm 0.08$ \\
DH-EP (pFF pCAB) & $0.071 \pm 0.012$ \\
DH-EPP (pFF pCAB) & $0.015 \pm 0.04$ \\
L-6 (pFF pCAB) & $0.083 \pm 0.015$ \\
M-6 (pFF pCAB) & $0.100 \pm 0.078$ \\
\hline
\end{tabular}

curve than the control DH5 $\alpha$ (pCDFpCAB). Simultaneously, as we expected, gross glucose consumption improved compared with L-6, increased from 16.2 to $20.4 \mathrm{~g} / \mathrm{L}$ (Figure 5A). The glucose consumption of all engineered strains changed obviously after every modification step. PHB titer of M-6 improved significantly, from 4.82 to $5.81 \mathrm{~g} / \mathrm{L}$ in comparison with that of L-6 (Figure 5D), and PHB content in M-6 strain reached 85.1 wt\% (Figure 5E). The only drawback was that $\mathrm{PHB}$ yield of M-6 reached $68.1 \%(\mathrm{~mol} / \mathrm{mol})$ slightly decreased compared with L-6 (Figure 5E). In general, compared with parental strain DH-EP (pFFpCAB), the PHB titer and content improved 41.71 and $24.41 \%$ in M-6, respectively. And compared with the control DH5 $\alpha$ (pCDFpCAB), the PHB content and yield of M-6 improved 61.9 and $141.7 \%$ in M-6, respectively (Figure 5). All the engineered strains produced some amount of acetate, M- 6 and L6 produced less acetate than DH-EP strain. We also calculated the acetate formation and glucose consumption normalized by residual cell mass (RCM).

In order to confirm that the glucose consumption rates in our engineered strains are indeed improved, we calculated the specific growth rate and glucose utilization rates of DH-EP (pFFpCAB), DH5 $\alpha$-EPP (pFFpCAB), L-6 (pFFpCAB), and M-6 (pFFpCAB). After $p t s G$ gene was deleted, the specific growth rate of $\mathrm{DH}$ EPP decreased from 0.20 to $0.09 \mathrm{~h}^{-1}$, and the glucose utilization rate decreased from 0.78 to $0.41 \mathrm{~g} \mathrm{~L}^{-1} \mathrm{~h}^{-1}$ (Table 4). After $g l f_{z m}$ integration based on L-6, the specific growth rate of M-6 (pFFpCAB) increased obviously from 0.28 to $0.50 \mathrm{~h}^{-1}$, improved $42.8 \%$ compared to that of $\mathrm{DH} 5 \alpha$ (pFFpCAB). The growth consumption rate recovered to $0.71 \mathrm{~g} \mathrm{~L}^{-1} \mathrm{~h}^{-1}, 73 \%$ higher than DH-EP (pFFpCAB). M-6 and L-6 showed lower normalized glucose consumption. Thus M-6 and L-6 had improved PHB biosynthesis and PHB productivity since they produced less byproduct acetate and consumed less glucose per residual cell mass (Figure 5F and Table 5).

We further examined the $\mathrm{CO}_{2}$ release from the constructed EP-bifido strains. The total $\mathrm{CO}_{2}$ release of $\mathrm{DH} 5 \alpha-\mathrm{EPP}$ (pFFpCAB) decreased to $32.5 \%$ compared to DH-EP (pFFpCAB) (Figure 6A). And the $\mathrm{CO}_{2}$ emission of L-6 (pFFpCAB), M-6 (pFFpCAB) improved 177 and 332\% compared to that of DH5 $\alpha$-EPP (pFFpCAB). We believe that the restoring growth contributed to the increased $\mathrm{CO}_{2}$ release. The $\mathrm{CO}_{2}$ yield of DH5 $\alpha$-EPP (pFFpCAB), L-6 (pFFpCAB), and M-6 (pFFpCAB) decreased to $16.9 \%(\mathrm{~mol} / \mathrm{mol}), 74.4 \%(\mathrm{~mol} / \mathrm{mol})$ and $92.4 \%$ ( $\mathrm{mol} / \mathrm{mol}$ ) compared to their controls, respectively (Figure 6B). The above data confirmed the recovery of growth after PTS system deficiency and the decreased $\mathrm{CO}_{2}$ emission from the L-6 (pFFpCAB) and M-6 (pFFpCAB) strains.

\section{DISCUSSION}

As environmental problems intensify, carbon saving or carbon sequestration pathways have become a new focus for biomanufacturing. Previously, we successfully constructed an efficient carbon-saving pathway in E. coli called the EP-bifido
A

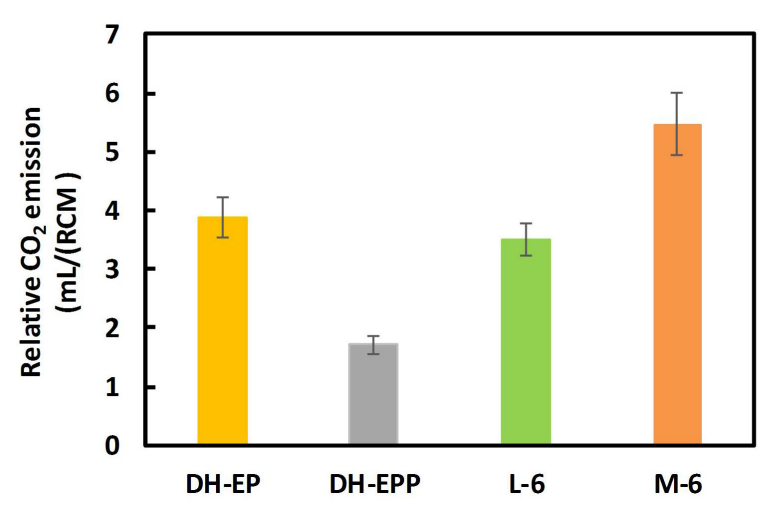

B

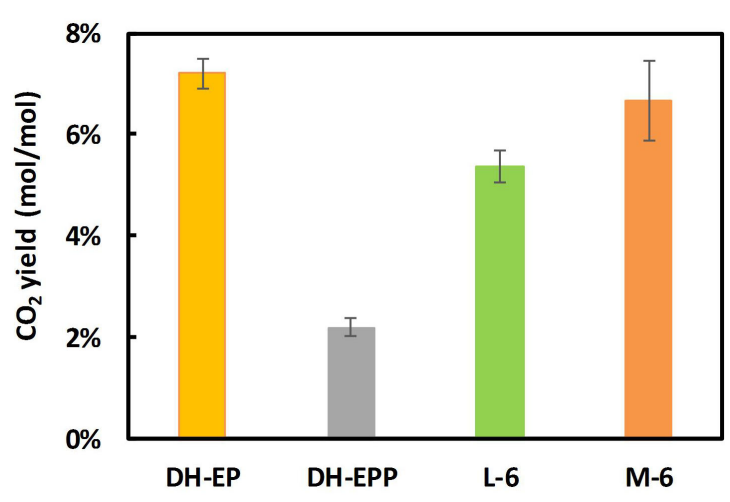

FIGURE 6 | Determination of $\mathrm{CO}_{2}$ release. (A) Relative $\mathrm{CO}_{2}$ emission and (B) $\mathrm{CO}_{2}$ yield. Strains were cultivated in modified M9 medium containing 20 g/L glucose at $37^{\circ} \mathrm{C}$ and $150 \mathrm{rpm} . \mathrm{CO}_{2}$ yield were calculated as $\mathrm{mol} / \mathrm{mol}$ glucose $\times 100 \%$. The experiments were performed in triplicate and error bars indicate s.d. 
pathway. This pathway has been applied to the production of several compounds that use acetyl-CoA as a precursor. As a degradable material, $\mathrm{PHB}$ has great application prospects. While we believe that there is potential for further optimization of $\mathrm{PHB}$ production by the EP-bifido pathway. In this study, we knocked out the $p t s G$ gene, a key glucose transporter of the PTS system that employs PEP as phosphate donor. The glucose consumption rate and cell growth were significantly reduced in the $p t s G$ mutant under aerobic fermentation conditions. The deficiency in the PTS system dramatically impairs glucose uptake and causes growth restriction. It is speculated that the reason for growth restriction is not insufficient glucose uptake, but the subsequent decrease in glucose phosphorylation efficiency due to limited glucokinase activity (Steinsiek and Bettenbrock, 2012).

To overcome this growth hindrance, we optimized the RBS sequences of galP and glk genes, encoding glucose permease and glucokinase, respectively. Subsequently, we introduced the Z. mobilis glucose transporter, $g l f_{z m}$, into the L- 6 high-yield strain, and observed cell growth recovery. After optimization, PHB yield reached $71.9 \%(\mathrm{~mol} / \mathrm{mol})$ in L-6 strain. In the resulting strain M-6, the intracellular PHB content reached $85.1 \mathrm{wt} \%$, and the titer reached $5.81 \mathrm{~g} / \mathrm{L}$. Previously, most studies have compensated for PTS knockout-induced inhibition of glucose uptake by overexpression of $g l k$, and $g a l P$ or by heterologous expression of $g l f$ (Snoep et al., 1994; Gosset, 2005; Lin et al., 2018). Instead, we applied MAGE technology to directly alter genomic glk and galP to optimize their expression (Gallagher et al., 2014). Meanwhile, the RBS library constructed using MAGE provided rich genotypes for subsequent screening of high-yield PHB strains. After $g l f_{z m}$ integration, recovered growth rate and glucose consumption was evident in strain M-6. Compared with parent strain DH-EP ( $\mathrm{pFFpCAB}$ ), the glucose consumption of M6 increased $4.6 \mathrm{~g} / \mathrm{L}, 29.1 \%$ higher than that of parent strain in Figure 5A. The PHB content and yield of strain M-6 improved compared with that of DH-EP(pFFpCAB). M-6 and L-6 showed lower normalized glucose consumption. Thus M-6 and L-6 had improved $\mathrm{PHB}$ production since they produced less by-product acetate and consumed less glucose per residual cell mass. We inferred that increased glucose uptake enhanced flux through

\section{REFERENCES}

Baba, T., Ara, T., Hasegawa, M., Takai, Y., Okumura, Y., Baba, M., et al. (2006). Construction of Escherichia coli K-12 in-frame, single-gene knockout mutants: the Keio collection. Mol. Syst. Biol. 2:2006.0008.

Bogorad, I. W., Lin, T. S., and Liao, J. C. (2013). Synthetic non-oxidative glycolysis enables complete carbon conservation. Nature 502, 693-697. doi: 10.1038/ nature 12575

Datsenko, K. A., and Wanner, B. L. (2000). One-step inactivation of chromosomal genes in Escherichia coli K-12 using PCR products. Proc. Natl. Acad. Sci. U.S.A. 97, 6640-6645. doi: 10.1073/pnas. 120163297

Erb, T. J., Berg, I. A., Brecht, V., Muller, M., Fuchs, G., and Alber, B. E. (2007). Synthesis of C5-dicarboxylic acids from C2-units involving crotonyl-CoA carboxylase/reductase: the ethylmalonyl-CoA pathway. Proc. Natl. Acad. Sci. U.S.A. 104, 10631-10636. doi: 10.1073/pnas.070279 1104

Gallagher, R. R., Li, Z., Lewis, A. O., and Isaacs, F. J. (2014). Rapid editing and evolution of bacterial genomes using libraries of synthetic DNA. Nat. Protoc. 9 , 2301-2316. doi: 10.1038/nprot.2014.082
EMP, which is supported by increased cell growth. The growth of DH-EP strain improved with decreased acetate formation in M-6 strain. The specific growth rate and $\mathrm{CO}_{2}$ release data of constructed strains further confirmed our inference. Our study provided an efficient way for improving glucose absorption and total carbon conversion rate in artificial carbon-saving pathways by replacing PTS with other glucose transporters. It also describes an efficient screening strategy for MAGE ssDNA recombineering technology. The efficient utilization of carbon sources has been one of the determinant for high productivity in microbial fermentation. In the future, the effective allocation of carbon resources and the construction of effective strategies for balancing cell growth and product biosynthesis will still be the direction of metabolic engineering.

\section{DATA AVAILABILITY STATEMENT}

All datasets generated for this study are included in the article/supplementary material.

\section{AUTHOR CONTRIBUTIONS}

QW and QQ designed the work. YLi, YLu, and JX performed the experiments. ZS and YLi analyzed the ${ }^{13} \mathrm{C}-\mathrm{MFA}$ data. QQ and $\mathrm{QL}$ encouraged this project. YLi and QW wrote the manuscript. All authors read and approved the final manuscript. All authors contributed to the article and approved the submitted version.

\section{FUNDING}

This work was supported by grants from the National Key R\&D Program of China (2019YFA0904900), the National Natural Science Foundation of China (31730003, 31670047, and 31770095), and Young Scholars Program of Shandong University and State Key Laboratory of Microbial Technology Open Projects Fund (M2019-11).
Gong, F., Cai, Z., and Li, Y. (2016). Synthetic biology for CO2 fixation. Sci. China Life Sci. 59, 1106-1114. doi: 10.1007/s11427-016-0304-2

Gosset, G. (2005). Improvement of Escherichia coli production strains by modification of the phosphoenolpyruvate:sugar phosphotransferase system. Microb. Cell Fact 4:14.

Haldimann, A., and Wanner, B. L. (2001). Conditional-replication, integration, excision, and retrieval plasmid-host systems for gene structure-function studies of bacteria. J. Bacteriol. 183, 6384-6393. doi: 10.1128/jb.183.21.6384-6393.2001

Hernandez-Montalvo, V., Martinez, A., Hernandez-Chavez, G., Bolivar, F., Valle, F., and Gosset, G. (2003). Expression of galP and glk in a Escherichia coli PTS mutant restores glucose transport and increases glycolytic flux to fermentation products. Biotechnol. Bioeng. 83, 687-694. doi: 10.1002/bit.10702

International Energy Agency (2019). Data From: Global Energy and $\mathrm{CO}_{2} \_$Status Report. Paris: International Energy Agency.

Kuhlman, T. E., and Cox, E. C. (2010). Site-specific chromosomal integration of large synthetic constructs. Nucleic Acids Res. 38:e92. doi: 10.1093/nar/gkp1193

Kyselova, L., Kreitmayer, D., Kremling, A., and Bettenbrock, K. (2018). Type and capacity of glucose transport influences succinate yield in two-stage cultivations. Microb. Cell Factor. 17:132. 
Lee, J. W., Na, D., Park, J. M., Lee, J., Choi, S., and Lee, S. Y. (2012). Systems metabolic engineering of microorganisms for natural and non-natural chemicals. Nat. Chem. Biol. 8, 536-546. doi: 10.1038/nchembio.970

Lee, S. J., Lee, D. Y., Kim, T. Y., Kim, B. H., Lee, J. W., and Lee, S. Y. (2005). Metabolic engineering of Escherichia coli for enhanced production of succinic acid, based on genome comparison and in silico gene knockout simulation. Appl. Environ. Microbiol. 71, 7880-7887. doi: 10.1128/aem.71.12.7880-7887. 2005

Lee, S. Y., and Choi, J. I. (2001). Production of microbial polyester by fermentation of recombinant microorganisms. Adv. Biochem. Eng. Biotechnol. 71, 183-207. doi: 10.1007/3-540-40021-4_6

Lee, S. Y., Lee, K. M., Chang, H. N., and Steinbuchel, A. (1994). Comparison of recombinant Escherichia-Coli strains for synthesis and accumulation of poly(3-hydroxybutyric acid) and morphological-changes. Biotechnol. Bioeng. 44, 1337-1347. doi: 10.1002/bit.260441110

Li, Y., Li, M., Zhang, X., Yang, P., Liang, Q., and Qi, Q. (2013). A novel wholephase succinate fermentation strategy with high volumetric productivity in engineered Escherichia coli. Bioresour. Technol. 149, 333-340. doi: 10.1016/j. biortech.2013.09.077

Li, Z. J., Cai, L., Wu, Q., and Chen, G. Q. (2009). Overexpression of NAD kinase in recombinant Escherichia coli harboring the phbCAB operon improves poly(3hydroxybutyrate) production. Appl. Microbiol. Biotechnol. 83, 939-947. doi: 10.1007/s00253-009-1943-6

Lin, P. P., Jaeger, A. J., Wu, T. Y., Xu, S. C., Lee, A. S., Gao, F. K., et al. (2018). Construction and evolution of an Escherichia coli strain relying on nonoxidative glycolysis for sugar catabolism. Proc. Natl. Acad. Sci. U.S.A. 115, 3538-3546. doi: 10.1073/pnas.1802191115

Meile, L., Rohr, L. M., Geissmann, T. A., Herensperger, M., and Teuber, M. (2001). Characterization of the D-xylulose 5-phosphate/Dfructose 6-phosphate phosphoketolase gene (xfp) from Bifidobacterium lactis. J. Bacteriol. 183, 2929-2936. doi: 10.1128/jb.183.9.2929-2936. 2001

Mozumder, M. S. I., De Wever, H., Volcke, E. I. P., and Garcia-Gonzalez, L. (2014). A robust fed-batch feeding strategy independent of the carbon source for optimal polyhydroxybutyrate production. Process Biochem. 49, 365-373. doi: 10.1016/j.procbio.2013.12.004

Posthuma, C. C., Bader, R., Engelmann, R., Postma, P. W., Hengstenberg, W., and Pouwels, P. H. (2002). Expression of the xylulose 5-phosphate phosphoketolase gene, xpkA, from Lactobacillus pentosus MD363 is induced by sugars that are fermented via the phosphoketolase pathway and is repressed by glucose mediated by CcpA and the mannose phosphoenolpyruvate phosphotransferase system. Appl. Environ. Microbiol. 68, 831-837. doi: 10.1128/aem.68.2.831-837. 2002

Ragauskas, A. J., Williams, C. K., Davison, B. H., Britovsek, G., Cairney, J., Eckert, C. A., et al. (2006). The path forward for biofuels and biomaterials. Science 311, 484-489. doi: 10.1126/science. 1114736
Schwander, T., Schada Von Borzyskowski, L., Burgener, S., Cortina, N. S., and Erb, T. J. (2016). A synthetic pathway for the fixation of carbon dioxide in vitro. Science 354, 900-904. doi: 10.1126/science.aah5237

Snoep, J. L., Arfman, N., Yomano, L. P., Fliege, R. K., Conway, T., and Ingram, L. O. (1994). Reconstruction of glucose uptake and phosphorylation in a glucose-negative mutant of Escherichia coli by using Zymomonas mobilis genes encoding the glucose facilitator protein and glucokinase. J. Bacteriol. 176, 2133-2135. doi: 10.1128/jb.176.7.2133-2135.1994

Steinsiek, S., and Bettenbrock, K. (2012). Glucose transport in Escherichia coli mutant strains with defects in sugar transport systems. J. Bacteriol. 194, 58975908. doi: 10.1128/jb.01502-12

Valle, F., Munoz, E., Ponce, E., Flores, N., and Bolivar, F. (1996). Basic and applied aspects of metabolic diversity: the phosphoenolpyruvate node. J. Industr. Microbiol. Biotechnol. 17, 458-462. doi: 10.1007/bf01574776

van der Walle, G. A., De Koning, G. J., Weusthuis, R. A., and Eggink, G. (2001). Properties, modifications and applications of biopolyesters. Adv. Biochem. Eng. Biotechnol. 71, 263-291. doi: 10.1007/3-540-40021-4_9

Wang, H. H., Isaacs, F. J., Carr, P. A., Sun, Z. Z., Xu, G., Forest, C. R., et al. (2009). Programming cells by multiplex genome engineering and accelerated evolution. Nature 460, 894-898. doi: 10.1038/nature08187

Wang, Q., Yu, H. M., Xia, Y. Z., Kang, Z., and Qi, Q. S. (2009). Complete PHB mobilization in Escherichia coli enhances the stress tolerance: a potential biotechnological application. Microb. Cell Factor. 8:47. doi: 10.1186/14752859-8-47

Wang, Q., Wu, C., Chen, T., Chen, X., and Zhao, X. (2006). Expression of galactose permease and pyruvate carboxylase in Escherichia coli ptsG mutant increases the growth rate and succinate yield under anaerobic conditions. Biotechnol. Lett. 28, 89-93. doi: 10.1007/s10529-005-4952-2

Wang, Q., Xia, Y. Z., Chen, Q., and Qi, Q. S. (2012). Incremental truncation of PHA synthases results in altered product specificity. Enzyme Microb. Technol. 50, 293-297. doi: 10.1016/j.enzmictec.2012.02.003

Wang, Q., Xu, J., Sun, Z., Luan, Y., Li, Y., Wang, J., et al. (2019). Engineering an in vivo EP-bifido pathway in Escherichia coli for high-yield acetyl-CoA generation with low $\mathrm{CO}_{2}$ emission. Metab. Eng. 51, 79-87. doi: 10.1016/j. ymben.2018.08.003

Conflict of Interest: The authors declare that the research was conducted in the absence of any commercial or financial relationships that could be construed as a potential conflict of interest.

Copyright (c) $2020 \mathrm{Li}$, Sun, Xu, Luan, Xu, Liang, Qi and Wang. This is an open-access article distributed under the terms of the Creative Commons Attribution License (CC BY). The use, distribution or reproduction in other forums is permitted, provided the original author(s) and the copyright owner(s) are credited and that the original publication in this journal is cited, in accordance with accepted academic practice. No use, distribution or reproduction is permitted which does not comply with these terms. 